\title{
Dual Extruder 3D-Bioprinter for Computer Designed Cardiac Structures
}

\author{
A.S. De la Nava ${ }^{1,2}$, A. Liberos ${ }^{1}$, E. G. Nieva ${ }^{1}$, I. Hernandez-Romero ${ }^{1}$, A. Simón ${ }^{1,2}$, M.E. Fernández- \\ Santos $^{1}$, F. Atienza ${ }^{1}$, A.M. Climent ${ }^{1}$, F. Fernandez-Aviles ${ }^{1}$ \\ ${ }^{1}$ Hospital GU Gregorio Marañón, IiSGM, CIBERCV, Madrid, Spain \\ ${ }^{2}$ Universidad Carlos III de Madrid, Spain
}

\begin{abstract}
A precise location of cells is of high interest for the characterization of cell interaction in in-vitro studies. Bioprinters can be a useful and automatized tool to produce these biological structures with precision.

The objective of this study is to develop and validate a low-cost novel dual-extruder $3 D$ bioprinter with enough resolution to study the electrophysiological interaction between different cell types. An open source classical 3D printer was adapted to incorporate two syringe extruders, allowing controlled delivery of hydrogel-cell ink. Four different parameters were studied to adjust precision: the needle lumen $(G)$, the density of the hydrogel (\%), the linear velocity of the extruder in the platform $(\mathrm{mm} / \mathrm{s})$ and the extrusion velocity (steps of the NEMA 17 extruder motor). Cell viability was tested with alamarBlue test and spatial distribution of cells was studied with fluorescence microscopy.

As a result of this study, thin lines of $1.18 \mathrm{~mm}$ in width with $85 \%$ viability after $24 \mathrm{~h}$ of printing process were obtained.
\end{abstract}

\section{Introduction}

Interaction between cardiomyocytes and fibroblasts is a key phenomenon to understand cardiac conduction in arrhythmias. However, classical in-vitro experiments do not allow a precise location of cells for the characterization of those cell interaction.

3D printing technologies represent a new field for biological application by reproducing a 3D environment for cell culture and proliferation with precision, combining different types of cells (1).

Recent studies present several procedures for producing and testing the type of hydrogel $(2,3)$ and the structure adaptation of conventional 3D plastic printers to $3 \mathrm{D}$ bioprinters. However, none of them present a software adaptation containing an algorithm for precision calibration and extruded value measurement.

The remaining of the paper contains the design of a Dual 3D-Bioprinter for computer designed cardiac structures with enough resolution to correctly position different cellular types to study the interaction between them.

\section{Materials and Methods}

\subsection{Adaptation of the bioprinter}

The open source classical plastic 3D-printer Prusa i3 Hephestos manufactured by BQ (Figure 1A) was adapted for biological component extrusion. This printer was originally designed by Josef Prusa containing three axes (X, Y and Z) that allow extrusion in Cartesian coordinates system of reference. To include the possibility of printing biological components, two identical replistruders designed by Hinton et al. (4) were added to the design, resulting in an extrusion mechanism bioprinter (Figure 1B). The replistruders were printed in PLA with a Makerbot 2.0 and transform the rotary motion, generated by NEMA 17 stepper motors, into a linear movement to push the syringe. The replistruder is composed of a small gear $(\mathrm{N}=13)$, large gear $(\mathrm{N}=52)$ and the syringe body. Additionally, a threated rod tighten with an elastic band completes the conversion of rotation movement into linear. The parts were polished to better adapt all the components in the system. To couple the replistruders to the $\mathrm{X}$ axis of the bioprinter, a rectangular base was designed (Figure 1C) using conventional CAD design programs and 3D printed with a Makerbot 2.0. Finally, the support structure was integrated in the bioprinter.

The printer was also equipped with PCB Heatedbed MK2a that allows for control temperature during the whole extrusion process.

\subsection{Cell Culture}

HL1 cardiac cell line can contract and can be serially passaged, maintaining their electrophysiological, biochemical and morphological properties (5).

The media used to culture the cell line was $87 \%$ Claycomb with a $10 \%$ of FBS, $1 \%$ PS, $1 \%$ Norepineprhine and $1 \%$ L-Glutamine. The media was changed twice a week and cell were grown at $37^{\circ} \mathrm{C}$ in an atmosphere of $5 \%$ $\mathrm{CO}_{2}$ and $95 \%$ air at a relative humidity of $95 \%$. The cells were first cultured in flask, i.e. two-dimensional cell 
cultures.

\subsection{Alginate Hydrogel}

To introduce the cells into the syringe of the replistruder, cells were embedded into an Alginate hydrogel. Alginate hydrogel was used as extracellular matrix because of its biocompatibility, ease of gelation and porosity of the matrix, that contributes to the diffusion of the nutrients (6).

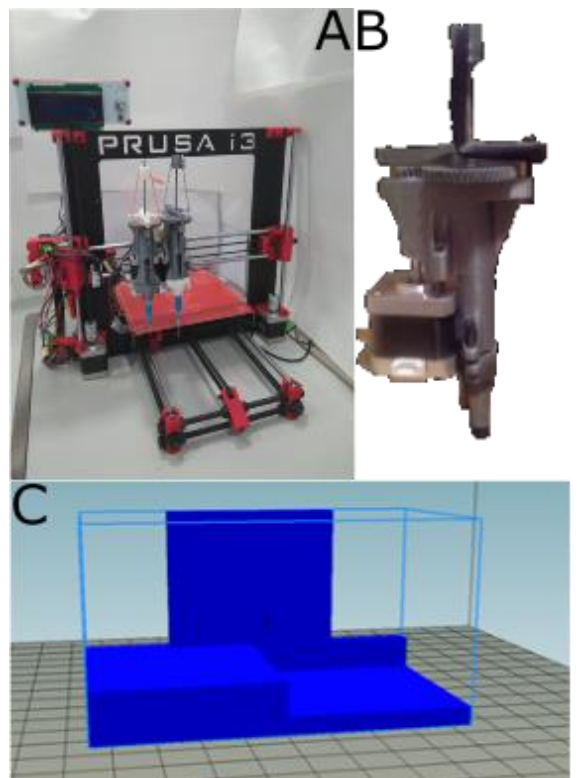

Figure 1. (A) Dual Extruder Bioprinter (B) Hinton extruder structure $(\mathrm{C}) \mathrm{CAD}$ model of the printed base to hold two extruders.

Two different Alginate concentrations were studied: 1.5 and $2 \%$ Alginate. The hydrogel was prepared by mixing 1.5 or $2 \%$ of Sodium Alginate powder (Tradissimo) with $50 \%$ PBS and $50 \%$ complete medium. The alginate was stirred and sterilized with UV light for 20 minutes. Then, cells were added and gently mixture with the hydrogel. A $0.1 \mathrm{M} \mathrm{CaCl}_{2}$ solution was used as cross linker and sprayed over the surface of the hydrogel to promote gelation and shape fidelity conservation after printing.

\subsection{Algorithm development}

The algorithm was developed based on the needle lumen $(\mathrm{G})$, the density of the hydrogel $(\%)$, the linear velocity of the extruder in the platform $(\mathrm{mm} / \mathrm{s})$ and the extrusion velocity (steps/s). Lines of $2 \mathrm{~cm}$ length were printed for each variable and width was measured. Cell viability was also tested with alamarBlue ${ }^{\circledR}$ reagent in each of the cases and spatial conformation of cells was studied with fluorescence techniques.

\subsubsection{Needle Lumen}

Needle diameter was tested to adjust the final precision of the extruder and the bioprinter: 4 different needles (Becton Dickinson) were tested to prove dependency of viability on syringe lumen: 16, 18, 21 and 26G. For smaller lumen, better precision is expected and relation of cell viability and needle lumen must be established.

Hydrogel was seeded and planted into a P96 well. 3 samples per type of lumen were incubated and alamarBlue $\AA$ tests were performed. The time of incubation of the cell culture with the alamarBlue ${ }^{\circledR}$ was 15 hours, to allow the reactant to equally diffuse over the hydrogel in the well.

\subsubsection{Alginate concentration and density- dependence study}

To quantify the relation between the steps of the stepper motor and the extruded value depending on density, the extrusion was made with three different liquids of variable density: water, water with salts and water with lipids, corresponding to a density of $1 \mathrm{~g} / \mathrm{ml}, 1.0892 \mathrm{~g} / \mathrm{ml}$ and $0.9124 \mathrm{~g} / \mathrm{ml}$ respectively.

Cell density was not studied for the algorithm development as the range of cell concentrations used are assumed not to change final density.

\subsubsection{Linear velocities and extrusion values}

Different values of linear velocities were tested to obtain its relation with the width of the printed line. The printing process was repeated for different velocities and extrusion values. The velocities tested ranged from 500 $\mathrm{mm} / \mathrm{s}$ to $3000 \mathrm{~mm} / \mathrm{s}$. The different extrusion values tested were: $18,22,26,30,34,38,42$ and 46 steps of the stepper motor coupled to the extruder.

\subsubsection{Viability tests}

To obtain a quantitative value of the viability of cells under the previous conditions, alamarBlue ${ }^{\circledR}$ (oxidationreduction colorimetric indicator) was used. Cells were cultured in a 96 well plate, 10000 cells per well was found to be the optimal cell concentration for correct development. $100 \pm 0.5 \mu \mathrm{l}$ of hydrogel were added per well and chemically cross-linked with a $0.1 \mathrm{M} \mathrm{CaCl}_{2}$ solution. Finally, $150 \pm 0.5 \mu \mathrm{l}$ of complete medium were added to each well. To prevent hydrogels from drying, the surrounding wells were filled with distilled water.

Absorbance for each well was measured using EMax ${ }^{\circledR}$ Plus Microplate Reader from bioNova cientifica. Measures were taken after 24 hours of incubation.

In addition, fluorescence microscopy was used to study the $3 \mathrm{D}$ conformational and spatial distribution of the cells 
in the hydrogel. Two different stains were used corresponding to different cell structures: CMFDA and permeable Propidium Iodine (PI). CMFDA was used to stain alive cells before introducing them into the hydrogel. For this purpose, $5 \mu \mathrm{l} / \mathrm{ml}$ of CMFDA (Thermo Fisher Scientific) stain was added to fresh medium containing the cells at a density of $5 \cdot 10^{6}$ cells $/ \mathrm{ml}$ and mixed for 1 hour at $37^{\circ} \mathrm{C}$ in darkness. Then, cells were washed with PBS to prevent excess of stain to expand in the hydrogel, centrifuged at $500 \mathrm{G}$ for five minutes and resuspended in desired concentration for hydrogel seeding. After 24 hours of seeding, PI was added in the same concentration and infused into the hydrogel for five minutes, allowing dead cells to become stained by the PI.

Cells in hydrogel were then analyzed with Leica DFC 310 FX Fluorescence Microscope.

\subsubsection{Software Implementation}

The communication with the printer is made by an Ardunio Mega 2560 board, that receives the delivery instructions (gcode) containing all the positions in Cartesian Coordinates. In addition, the gcode included the extrusion values for each segment (i.e. the number of steps the stepper motor in the extruder needed to for a given segment of the structure to be printed). Conventional slicer programs for 3D printing do not contemplate a specific algorithm for biological components and, as a result, the extrusion value needs to be modified. The developed algorithm included all the parameters studied in this paper, and implemented viable solutions for correct printing.

Once behavior of the fluids in the syringe was studied, a Matlab program was designed to produce the gcode necessary to print the structure in regular 3D printing programs. The Matlab program was designed with the algorithm obtained during the experiments. As an input, the program received the density of the fluid and the shape of the structure to be extruded. Finally, an extra option was added in case the user wanted to design its own model.

\section{Results}

\subsection{Needle lumen}

Results were observed in all the wells: cells were adhering to the hydrogel and survived in all the cases. The needle lumen affects the spatial configuration of the cells in the hydrogel. AlamarBlue ${ }^{\circledR}$ test results demonstrate higher proliferation rates for cells extruded with the $26 \mathrm{G}$ needle, corresponding to a final $83,67 \%$ of proliferation rate. Finally, the proliferation rates for the $21 \mathrm{G}, 18 \mathrm{G}$ and 16G were $44.15 \%, 65.52 \%$ and $45.58 \%$ respectively. For the spatial spreading of the cells, higher spreading rates were obtained for higher lumen value.

\subsection{Alginate concentration and density- dependence study}

Viability tests showed that $2 \%$ Alginate solution was optimum for cell development and proliferation. Shape fidelity studies demonstrated consistency of the atmosphere-hydrogel barrier by conserving shape Furthermore, fusiform cells were observed in the hydrogel after 24 hours of implantation. 2\% Alginate hydrogel provides enough a sustenance matrix to get attached to the hydrogel allowing diffusion of nutrients over the matrix.

Regarding, density-dependence study, linear behavior was observed for the dependence on density. For higher density, higher number of steps were needed to extrude a given volume.

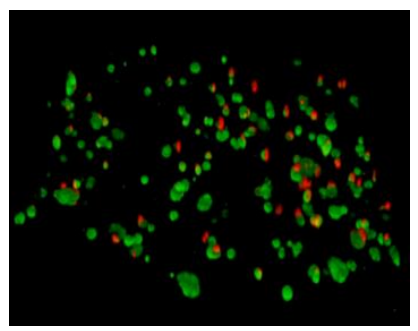

Figure $2.85 \%$ viability after 24 hours of incubation of cells in hydrogel died with CMFDA and PI.

\subsection{Extrusion velocity}

A relation for the linear velocity was stablished: fthe higher velocities, width of the printed lines resulted in higher values than the width for lower velocities, despite the same volumes were extruded. As expected, higher extrusion values, resulted in thicker lines (Figure 3). It must be pointed out that for very low extruded values (18 steps), lines were printed discontinuously on the Petri dish. The velocity at which lowest width and complete lines were drawn was found to be $500 \mathrm{~mm} / \mathrm{s}$ with a final width of $1.18 \mathrm{~mm}$.

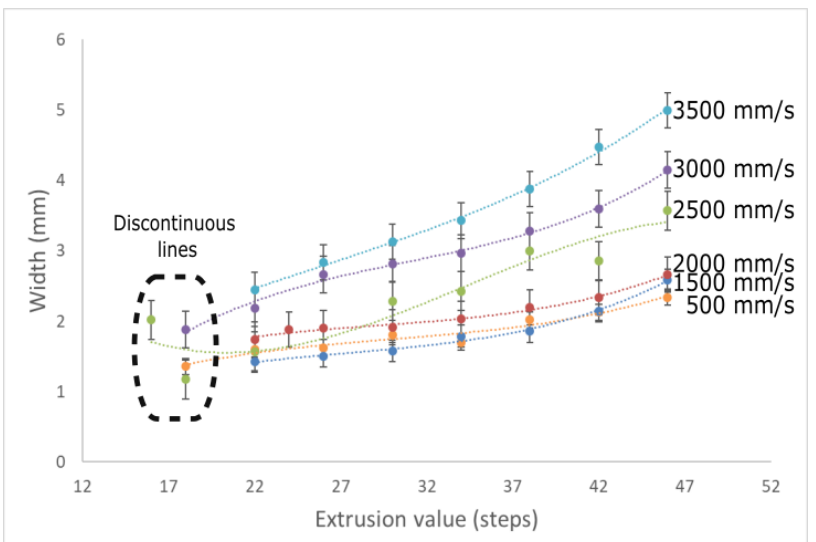

Figure 3. Width value (mm) vs. Extrusion value (steps) at different deposition velocities. 


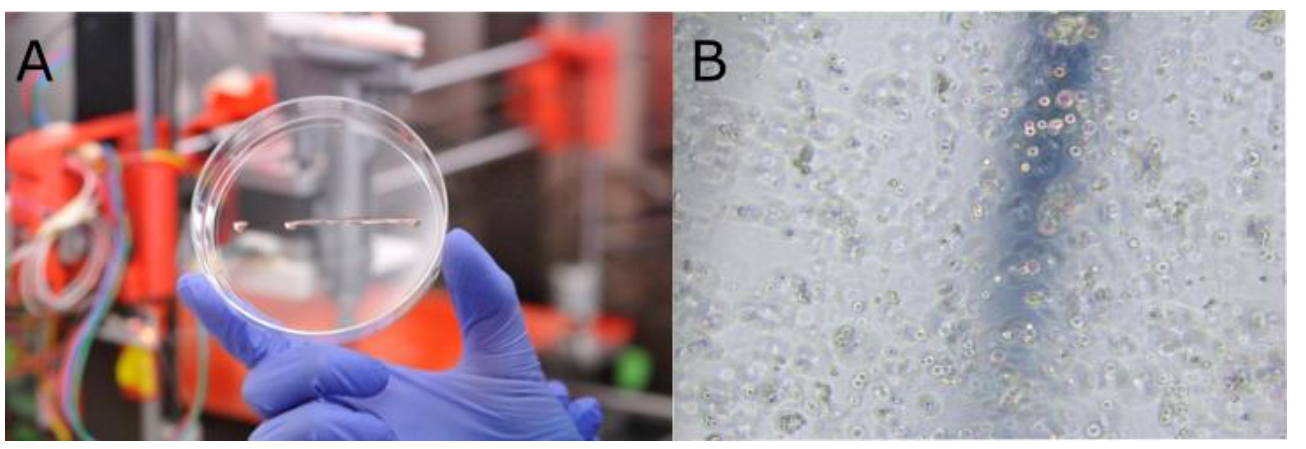

Figure 4. (A) Final bioprinted line with a width of $1.18 \mathrm{~mm}$.

bution printed structure.

\subsection{Software Implementation}

The final software contained different options. First, the type of shape to be printed can be designed by the user. The next implementation resided on the type of hydrogel used and the density of it. Finally, the extrusion value was calculated based on the estimations in Figure 3. The code completely implements the geometry and sends the gcode to Repetier, the software used to turn on and print with the Prusa i3 3D printer.

The final printed lines ensured a minimum resolution of $1.18 \mathrm{~mm}$ (Figure $4 \mathrm{~A}$ ) in width with a viability of $85 \%$ after 24 hours of incubation (Figure 2). Printed lines presented a homogeneous distribution of the cells over the hydrogel ensuring encapsulation and shape fidelity (Figure 4B).

\section{Discussion}

A totally functional 3D Bioprinter for two different Cardiac Structures was implemented, ensuring a precise deposition of different cell types for in vitro studies.

A new algorithm was developed de novo and generalized to be applied to any hydrogel for HL1 cells. In addition, a user-friendly interface was created for nonexpert users to print with the bioprinter.

The use of the algorithm integrated in the Matlab interface provides a new tool to rapidly program the extrusion of biological components obtaining a known extruded volume and known resolution.

The 3D Bioprinter has a direct application on research fields, to prove and study behavior of different cell types in in vitro conditions. In addition, the use of the algorithm for calibration and printing process ensures minimum known resolution values for printer using the extruder designed by Hinton et al. (4).

The implementation and development of this technology opens new research lines to understand and study the interaction of cells in vitro and therefore, developing new methods to mimic the cardiac tissue with different cells types in the laboratory, approaching to the artificial production of heart tissue and heart structures.

\section{Acknowledgements}

This work has been posible thanks to the financing ofthe Instituto de Salud Carlos III, Ministerio de Ciencia e Innovación (PI13-01882, PI13-00903, PI14/00857, and IJCI-2014- 22178), Sociedad Española de Cardiología [Ayudas a la Investigación Clínica 2015]; Ministerio de Ciencia e Innovación (Red RIC RD12.0042.0001) and cofinanced with FEDER funds.

\section{References}

1. Fisher MB, Mauck RL. Tissue Engineering and Regenerative Medicine: Recent Innovations and the Transition to Translation. Tissue Eng Part B Rev [Internet]. 2013 Feb 18;19(1):1-13.

2. Hoffman AS. Hydrogels for biomedical applications. 2002;54:3-12.

3. Merceron TK, Murphy S V. Hydrogels for 3D Bioprinting Applications. Essentials of 3D Biofabrication and Translation. Elsevier Inc.; 2015. 249$270 \mathrm{p}$.

4. Hinton TJ, Jallerat Q, Palchesko RN, Park JH, Grodzicki MS, Shue H-J, et al. Three-dimensional printing of complex biological structures by freeform reversible embedding of suspended hydrogels. Sci Adv. 2015;1(9):e1500758-e1500758.

5. Claycomb WC, Lanson NAJ, Stallworth BS, Egeland DB, Delcarpio JB, Bahinski A, et al. HL-1 cells: a cardiac muscle cell line that contracts and retains phenotypic characteristics of the adult cardiomyocyte. Proc Natl Acad Sci U S A. 1998 Mar;95(6):2979-84.

6. Jia J, Richards DJ, Pollard S, Tan Y, Rodriguez J, Visconti RP, et al. Engineering alginate as bioink for bioprinting. Acta Biomater. 2014 Oct;10(10):4323-31.

Address for correspondence.

Andreu M. Climent

Laboratorio de órganos y matrices bioartificiales

Instituto de Investigación Sanitaria Gregorio Marañón

Hospital GU Gregorio Marañón. Edificio Materno Infantil.

cl O'Donnell 48, 28009, Madrid, Spain

andreu.climent@gmail.com 